\title{
Dentigerous Cystic Changes in the Follicles Associated with Radiographically Normal Impacted Mandibular Third Molars
}

\author{
Ashok Dongol (D), ${ }^{1}$ Alok Sagtani ${ }^{D},{ }^{2}$ Mehul Rajesh Jaisani ${ }^{(D},{ }^{1}$ Arpita Singh, ${ }^{3}$ \\ Ashish Shrestha $\mathbb{D}^{4}{ }^{4}$ Anju Pradhan, ${ }^{5}$ Pradeep Acharya, ${ }^{1}$ Anjani Kumar Yadav, ${ }^{1}$ \\ Ram Prasad Yadav, ${ }^{1}$ Arun Kumar Mahat, ${ }^{1}$ Iccha Kumar Maharjan, ${ }^{6}$ and Leeza Pradhan ${ }^{7}$ \\ ${ }^{1}$ Department of Oral and Maxillofacial Surgery, BP Koirala Institute of Health Sciences, Dharan, Nepal \\ ${ }^{2}$ Patan Academy of Health Sciences, Kathmandu, Nepal \\ ${ }^{3} B$ \& $C$ Hospital, Birtamod, Nepal \\ ${ }^{4}$ Department of Oral Pathology, BP Koirala Institute of Health Sciences, Dharan, Nepal \\ ${ }^{5}$ Department of Pathology, BP Koirala Institute of Health Sciences, Dharan, Nepal \\ ${ }^{6}$ Department of Oral Medicine and Radiology, BP Koirala Institute of Health Sciences, Dharan, Nepal \\ ${ }^{7}$ Kathmandu Model Hospital, Kathmandu, Nepal
}

Correspondence should be addressed to Ashok Dongol; ashokdongol@hotmail.com

Received 19 October 2017; Accepted 12 February 2018; Published 20 March 2018

Academic Editor: Giuseppina Campisi

Copyright (C) 2018 Ashok Dongol et al. This is an open access article distributed under the Creative Commons Attribution License, which permits unrestricted use, distribution, and reproduction in any medium, provided the original work is properly cited.

Objective. To assess the incidence of dentigerous cystic changes in the follicles of radiographically normal impacted mandibular third molars. Methods. One hundred and thirteen follicles obtained after surgical removal of impacted mandibular third molars with radiolucency of less than $2.5 \mathrm{~mm}$ in the radiograph were sent for histopathologic evaluation to evaluate pathologic changes. Results. The incidence of dentigerous cystic changes observed was $15.9 \%$, that is, 18 out of 113 patients (51 males and 62 females), with the maximum incidence of cystic changes seen in the follicular space size of $0.5 \mathrm{~mm}$. The mean age of the patients included was $27.8 \pm 8.1$. The most common indication for extraction among the patients in this study was recurrent pericoronitis (95\%). There were no statistically significant differences in occurrence of cystic changes based on age, gender, angulation, relation to ramus, depth, side of impaction, and follicle size $(P>0.05)$. Conclusion. Dental follicles obtained from surgically removed impacted mandibular third molars should be submitted for histopathologic examination irrespective of the radiographic size of the follicle.

\section{Introduction}

Impacted mandibular third molars are common among patients seen in the oral and maxillofacial surgery [1]. Infection, nonrestorable caries, cysts, tumors, and destruction of the adjacent teeth and bone are considered as indications for the removal of impacted third molars [2]. However, prophylactic extraction of the asymptomatic impacted third molar is routinely practiced in Europe and the United States. The justification for prophylactic extraction includes the need to minimize the risk of pathologic changes like cysts and tumors, increased difficulty of surgery with age, and reduction of the risk of mandibular angle fracture, and that the third molars have no definite role in the mouth [3]. The prophylactic extraction is still controversial.

The dental follicle is an ectomesenchymal tissue that surrounds the developing tooth germ. In a radiograph, it is seen as a normal homogeneous radiolucent space around the crown of a developing tooth and is known as the follicular space. The dental follicle associated with the impacted third molar has the potential to undergo cystic degeneration and form dentigerous cyst, odontogenic keratocyst, and ameloblastoma [4]. Follicular space of size less than $2.5 \mathrm{~mm}$ in width is considered as radiographically normal or not being associated with any pathology [5]. However, this may not be a reliable method to know the pathology associated with 
impacted teeth. So, it would be prudent to do a histological examination of the follicle associated with the impacted teeth as the absence of radiographic disease may not necessarily indicate the absence of the disease. The incidence of the pathological changes associated with impacted teeth seems to be higher than that reported from the radiographic studies [4, 6-9]. The removal of impacted teeth followed by the histopathologic examination of the follicle associated can thus decrease the chances of development of cysts and tumors and alleviate extensive treatment [3].

Therefore, the present study was conducted to assess the incidence of pathologic changes in the follicle of radiographically normal impacted mandibular third molars and to evaluate the association of cystic changes in the follicle with the patient's age, sex, angular position, and contact of the impacted tooth with adjacent tooth.

\section{Materials and Methods}

One hundred and thirteen patients aged 16-60 years with impacted mandibular third molars with a follicular space of less than $2.5 \mathrm{~mm}$ in the panoramic radiograph were included in the study. For radiographic measurement of the follicular size, contours of the impacted mandibular third molar and pericoronal radiolucency were traced on the tracing paper on the $\mathrm{X}$-ray viewer. A straight line $\left(\mathrm{AA}^{\prime}\right)$ passing through the long axis of the impacted tooth was drawn, and the other line $\left(\mathrm{BB}^{\prime}\right)$ was drawn perpendicular to $\mathrm{AA}^{\prime}$ and passing through the centre of the crown. The widest point of the follicular space was measured as per the method reported by Damante and Fleury [10]. Another straight line $\left(\mathrm{CC}^{\prime}\right)$ was drawn from the intersection of the two lines $\left(\mathrm{AA}^{\prime}\right.$ and $\left.\mathrm{BB}^{\prime}\right)$ to the widest area of the follicular space. Along this line $\left(\mathrm{CC}^{\prime}\right)$, the follicle size was determined by measuring the distance between the contour of the tooth and the widest area of the follicle using a caliper ruler (Figure 1).

After complete extraction of the tooth, follicular tissue around the crown of the tooth was collected in $10 \%$ neutral buffered formalin and sent for routine histopathological examination and stained with hematoxylin and eosin. One hundred and thirteen follicles thus obtained were sent for histopathologic evaluation to evaluate pathologic changes. A pericoronal radiolucency with a histopathologically continuous lining of the nonkeratinized stratified squamous epithelium and a cystic space between enamel and overlying tissue was considered as a dentigerous cyst (Figure 2), whereas tissue devoid of epithelial lining with no epithelial cell rests was considered as the normal follicle (Figure 3).

The association between the follicle and pathologic changes, age, gender, angular position, and contact of the impacted tooth with adjacent tooth was statistically evaluated. The measurement of association of categorical variables was done through the chi-square test and calculation of odds ratio with $95 \%$ confidence interval.

The study was conducted in accordance with the Declaration of Helsinki. Ethical approval was obtained from the Institutional Ethical Review Board, BP Koirala Institute of Health Sciences. Informed written consent was taken from the patients.

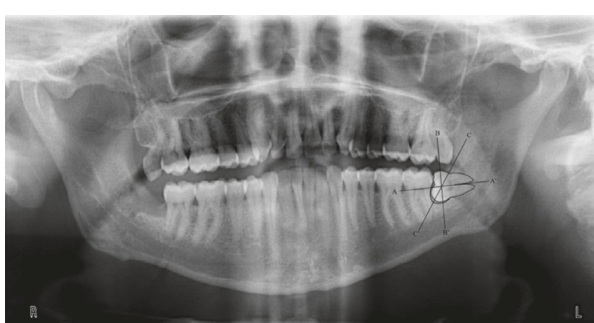

FIgURE 1: Follicle size measurement.

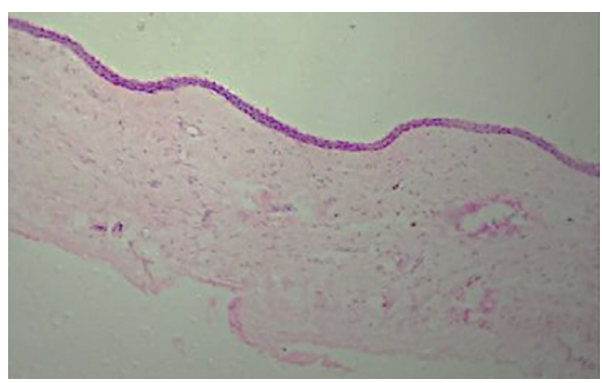

Figure 2: Lining of the nonkeratinized stratified squamous epithelium with odontogenic islands suggestive of dentigerous cyst (H\&E stains; 10x).

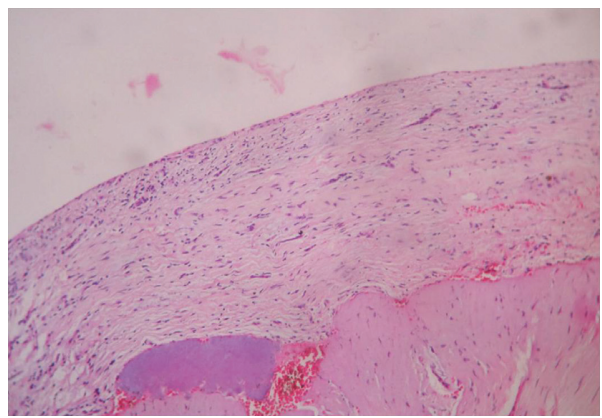

Figure 3: Dental follicle devoid of epithelial lining (H\&E stains; 10x).

\section{Results}

Of the 113 follicle tissues studied, 51 (45.1\%) were from male patients and $62(54.9 \%)$ from female patients. The age of the patients ranged from 16 to 53 years with the maximum number of patients in the range between 26 and $30(n=32)$ followed by 21 - to 25 -year $(n=30)$ age group. The mean age of the patients was 27.8 years with the standard deviation of \pm 8.1 . The most common cause of extraction of the impacted mandibular third molar was recurrent pericoronitis which was seen in 95 patients $(84.1 \%)$, followed by carious third molar and carious second molar in 7 patients each (6.2\%) and prophylactic in 4 patients (3.5\%). Normal follicle (69 patients, $61.1 \%$ ) was the most common histopathologic finding with only 18 patients (15.9\%) showing dentigerous cyst, as shown in Figure 4. Considering the normal follicle, inflammatory tissue, and granulation tissue as noncystic changes, 18 (15.9\%) had cystic changes and 95 (84.1\%) showed no cystic changes which is suggestive of less incidence of cystic changes in the follicle around the impacted third molar (Figure 5). 


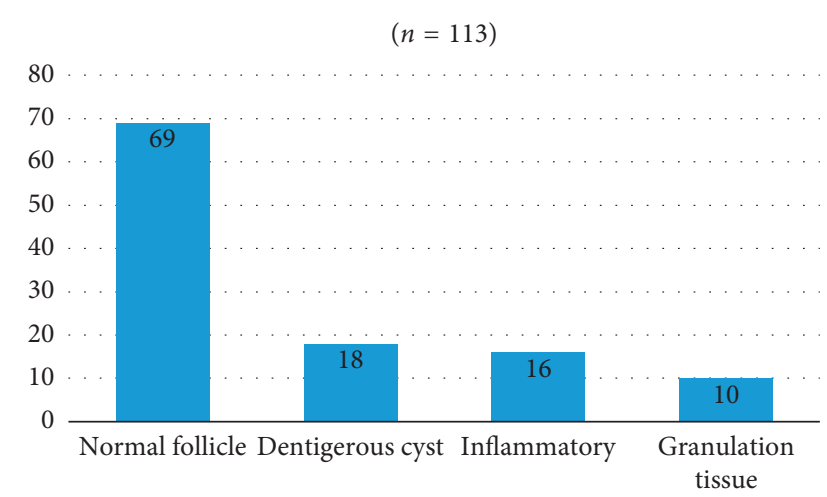

Figure 4: Frequency distribution of histopathological diagnosis.

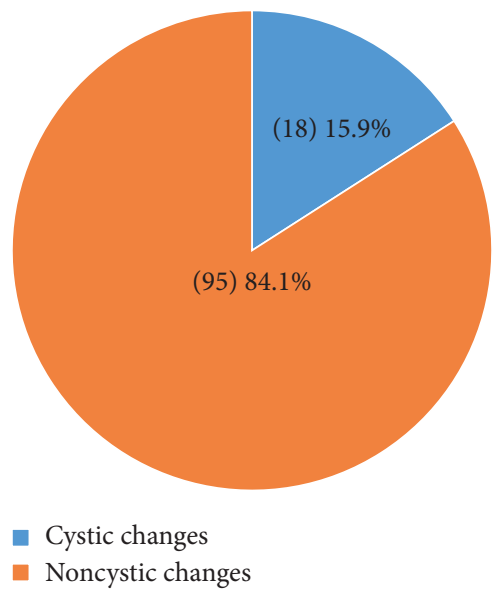

FIGURE 5: Frequency distribution of cystic changes.

There was no statistically significant difference $(P=0.95)$ in the occurrence of cystic changes based on gender. Ten out of 62 female patients and 8 out of 51 male patients had cystic changes. There was no statistically significant difference in the occurrence of cystic changes among the patients in relation to age (Table 1). Cystic changes were seen in tissues from patients as young as 16 and as old as 42, and the mean age of the patients with cystic changes was found to be 27 years.

No statistical significance was observed in the occurrence of cystic changes based on the angulation pattern of the impacted tooth $(P=0.816)$, the depth of impaction $(P=0.826)$, and relationship of the impacted tooth with the ramus $(P=0.442)$, on side distribution of the impacted mandibular third molar as shown in Table 2.

Upon evaluation of association of dentigerous cystic changes with the follicle size, cystic changes were observed more in the follicle size of $0.5 \mathrm{~mm}$ as shown in Table 3. No statistically significant difference was found in the occurrence of cystic changes based on the follicle size (Table 4).

\section{Discussion}

Impacted mandibular third molars are routinely indicated for extraction when signs and symptoms of pathosis are present. But there is still no general agreement on whether to remove asymptomatic third molars or not. As the incidence of systemic diseases, pathological conditions associated with the impacted tooth, and postsurgical morbidity increases with age, so do the difficulty and complications of impacted third molar surgery [3]. These causes support the prophylactic removal. Proponents of prophylactic removal have also suggested high incidence of cystic changes associated with impacted third molars with the follicular space even less than $2.5 \mathrm{~mm}$, which is considered radiographically normal [5]. Baykul et al. [6] found 50\%, Saravana and Subhashraj [7] found 46\%, Glosser and Campbell [8] found 37\%, Rakprasitkul [4] found 35\%, Adelsperger et al. [9] found 34\%, and Yildırım et al. [11] found $23 \%$ dentigerous cystic changes in impacted third molars. Besides dentigerous cysts as the detected pathologic entity, others have also reported odontogenic keratocyst, calcifying odontogenic cysts, ameloblastoma, myxoma, and odontogenic fibromas [12-15].

Therefore, the present study was carried out to evaluate the pathologic changes associated with the normal radiographic follicular size in mandibular impacted third molars. Glosser and Campbell [8] and Curran et al. [12] suggested that any follicle with the stratified squamous epithelium should be regarded as a dentigerous cyst. Cystic changes observed in our study were only of dentigerous cyst with the nonkeratinized stratified squamous epithelium in $15.9 \%$ of the follicles. The value observed in the present study is lower than those reported by the aforementioned authors. But still, this observation should alert the surgeons to submit the follicles obtained after extraction for histopathologic evaluation. Less percentage of cystic changes in this study is probably because the pathologic condition may have involuted and not progressed to detectable lesion as the mean age of the patients is higher than that compared to other studies. So with age-related changes, the tissue could have undergone conversion to a quiescent state, persisting only as a histologic aberration of little clinical significance [16]. Thus, the patients with cystic changes should be kept for long-term follow-up to assess the progression of the disease. This will eventually help in the early detection of pathology if any related to cystic changes followed by its management and thus may prevent morbidities associated with the cyst. Also, the added cost of diagnosis based on the pathologic evaluation is worth than the risk of future deterioration from cystic changes.

The mean age of the patients with cystic changes was found to be 27 years in our study with the occurrence of cystic changes from 16 years to 42 years of age. Studies have suggested that groups older than the second decade show higher incidences of pathologic changes $[8,9]$. Histopathologic diagnosis of cystic changes showed a female-to-male ratio of 1.25:1 in our study considering the fact that the incidence of impaction is more in females [17]. Other studies have observed male predominance with cystic changes, but the reason for this gender difference is still unknown $[4,9,18]$. The most common cause for extraction among the patients in this study was recurrent pericoronitis (84.1\%). In the context of our country, patients rarely seek dental treatment when the tooth is asymptomatic. The alarming symptoms of acute and/or recurrent pericoronitis might be 
TABLE 1: Relationship between dentigerous cystic changes and age.

\begin{tabular}{lcccccc}
\hline Sociodemographic characteristics & Cystic changes & Noncystic changes & $t$-test & Lower 95\% CI & Upper 95\% CI & $P$ value \\
\hline Age (years) & $27 \pm 7.4$ & $27.9 \pm 8.3$ & 0.44 & -5.08 & 3.23 & 0.66 \\
\hline
\end{tabular}

TABLE 2: Relationship between dentigerous cystic changes and angulation, depth, and relation of the impacted tooth to ramus.

\begin{tabular}{|c|c|c|c|c|c|}
\hline \multirow{2}{*}{ Parameters related to impaction } & & \multirow{2}{*}{$\begin{array}{c}\text { Number } \\
(n=113)\end{array}$} & \multicolumn{2}{|c|}{ Dentigerous cyst } & \multirow{2}{*}{$P$ value } \\
\hline & & & Present & Absent & \\
\hline \multirow{4}{*}{ Angulation of the impacted third molar } & Mesioangular & 52 & $7(13.5 \%)$ & $45(86.5 \%)$ & \multirow{4}{*}{0.816} \\
\hline & Horizontal & 25 & $4(16 \%)$ & $21(84 \%)$ & \\
\hline & Vertical & 28 & $6(21.4 \%)$ & $22(78.6 \%)$ & \\
\hline & Distoangular & 8 & $1(12.5 \%)$ & $7(87.5 \%)$ & \\
\hline \multirow{3}{*}{ Depth of impaction } & Position A & 37 & $5(13.5 \%)$ & $32(86.5 \%)$ & \multirow{3}{*}{0.826} \\
\hline & Position B & 68 & $12(17.6 \%)$ & $56(82.4 \%)$ & \\
\hline & Position C & 8 & $1(12.5 \%)$ & $7(87.5 \%)$ & \\
\hline \multirow{3}{*}{ Relationship of the impacted tooth with ramus } & Class I & 41 & $7(17.1 \%)$ & $34(82.9 \%)$ & \multirow{3}{*}{0.442} \\
\hline & Class II & 64 & $11(17.2 \%)$ & $53(82.8 \%)$ & \\
\hline & Class III & 8 & $0(0 \%)$ & $8(100 \%)$ & \\
\hline \multirow{2}{*}{ Side distribution } & Left & 51 & $9(17.6 \%)$ & $42(82.4 \%)$ & \multirow{2}{*}{0.65} \\
\hline & Right & 62 & $9(14.5 \%)$ & $53(85.5 \%)$ & \\
\hline
\end{tabular}

TABLe 3: Incidence of dentigerous cyst according to the follicular size.

\begin{tabular}{lcc}
\hline Follicular size $(\mathrm{mm})$ & Number of patients $(n=113)$ & Dentigerous cyst \\
\hline 0.5 & 12 & $3(25 \%)$ \\
1 & 45 & $7(15.5 \%)$ \\
1.5 & 18 & $2(11.1 \%)$ \\
2 & 28 & $4(14.3 \%)$ \\
2.5 & 10 & $2(20 \%)$ \\
\hline
\end{tabular}

TABle 4: Relationship between dentigerous cystic changes and follicle size.

\begin{tabular}{lcccccc}
\hline & Cystic changes & Noncystic changes & $t$-test & Lower 95\% CI & Upper 95\% CI & $P$ value \\
\hline Follicle size $(\mathrm{mm})$ & $1.36 \pm 0.66$ & $1.42 \pm 0.58$ & 3.58 & -0.36 & 0.25 & 0.72 \\
\hline
\end{tabular}

the convincing reason for the patients to seek for immediate dental treatment and be motivated for extraction.

A significant difference could not be found regarding the relationship between dentigerous cystic changes and depth of impaction as well as the relation of the impacted third molar with ramus in our study. Although no statistically significant association between cystic changes and angulation of the third molar was observed, higher probability of cystic changes was found in vertically positioned impacted third molars (21.4\%) as observed in the study by Baykul et al. [6]. However, Knutsson et al. [17] and Eliasson et al. [5] reported higher incidence of pathologic changes in horizontally positioned impacted third molars, while Adaki et al. [16] found higher probability of cystic changes in the distoangular impaction. Such differences observed may be due to different study designs with different inclusion criteria. But the variation also suggests that all types of the angulation pattern of impaction can potentially be associated with cystic changes.

\section{Conclusion}

Cystic changes may be found in small follicular spaces, whereas there may be histologically normal tissues in big radiolucent lesions [19]. Therefore, according to the results of this study, dental follicles of surgically removed impacted third molars should be submitted for histopathologic examination irrespective of the follicle size because dentigerous cystic changes occurring in the follicle tissue cannot be disregarded. There should also be a standard follow-up protocol for periodic evaluation of the patients with evidence of cystic changes to detect any further clinical or pathologic changes so as to prevent morbidity associated with it.

\section{Conflicts of Interest}

The authors declare that they have no conflicts of interest.

\section{References}

[1] R. D. Marciani, "Third molar removal: an overview of indications, imaging, evaluation, and assessment of risk," Oral and Maxillofacial Surgery Clinics of North America, vol. 19, no. 1, pp. 1-13, 2007.

[2] P. Stathopoulos, M. Mezitis, C. Kappatos, S. Titsinides, and E. Stylogianni, "Cysts and tumors associated with impacted third molars: is prophylactic removal justified?," Journal of 
Oral and Maxillofacial Surgery, vol. 69, no. 2, pp. 405-408, 2011.

[3] W. L. Adeyemo, "Do pathologies associated with impacted lower third molars justify prophylactic removal? A critical review of the literature," Oral Surgery, Oral Medicine, Oral Pathology, Oral Radiology, and Endodontology, vol. 102, no. 4, pp. 448-452, 2006.

[4] S. Rakprasitkul, "Pathologic changes in the pericoronal tissues of unerupted third molars," Quintessence International, vol. 32, no. 8, pp. 633-638, 2001.

[5] S. Eliasson, A. Heimdahl, and ^. Nordenram, "Pathological changes related to long-term impaction of third molars," International Journal of Oral and Maxillofacial Surgery, vol. 18, no. 4, pp. 210-212, 1989.

[6] T. Baykul, A. A. Saglam, U. Aydin, and K. Başak, "Incidence of cystic changes in radiographically normal impacted lower third molar follicles," Oral Surgery, Oral Medicine, Oral Pathology, Oral Radiology, and Endodontology, vol. 99, no. 5, pp. 542-545, 2005.

[7] G. H. L. Saravana and K. Subhashraj, "Cystic changes in dental follicle associated with radiographically normal impacted mandibular third molar," British Journal of Oral and Maxillofacial Surgery, vol. 46, no. 7, pp. 552-553, 2008.

[8] J. W. Glosser and J. H. Campbell, "Pathologic change in soft tissues associated with radiographically 'normal' third molar impactions," British Journal of Oral and Maxillofacial Surgery, vol. 37, no. 4, pp. 259-260, 1999.

[9] J. Adelsperger, J. H. Campbell, D. B. Coates, D.-J. Summerlin, and C. E. Tomich, "Early soft tissue pathosis associated with impacted third molars without pericoronal radiolucency," Oral Surgery, Oral Medicine, Oral Pathology, Oral Radiology, and Endodontology, vol. 89, no. 4, pp. 402-406, 2000.

[10] J. H. Damante and R. N. Fleury, "A contribution to the diagnosis of the small dentigerous cyst or the paradental cyst," Pesquisa Odontológica Brasileira, vol. 15, no. 3, pp. 238-246, 2001.

[11] G. Yıldırım, H. Ataoğlu, A. Mihmanlı, D. Kızıloğlu, and M. C. Avunduk, "Pathologic changes in soft tissues associated with asymptomatic impacted third molars," Oral Surgery, Oral Medicine, Oral Pathology, Oral Radiology, and Endodontology, vol. 106, no. 1, pp. 14-18, 2008.

[12] A. E. Curran, D. D. Damm, and J. F. Drummond, "Pathologically significant pericoronal lesions in adults: histopathologic evaluation," Journal of Oral and Maxillofacial Surgery, vol. 60, no. 6, pp. 613-617, discussion 618, 2002.

[13] V. Kotrashetti, A. Kale, S. Bhalaerao, and S. Hallikeremath, "Histopathologic changes in soft tissue associated with radiographically normal impacted third molars," Indian Journal of Dental Research, vol. 21, no. 3, p. 385, 2010.

[14] A. H. Mesgarzadeh, H. Esmailzadeh, M. Abdolrahimi, and M. Shahamfar, "Pathosis associated with radiographically normal follicular tissues in third molar impactions: a clinicopathological study," Indian Journal of Dental Research, vol. 19, no. 3, pp. 208-212, 2008.

[15] E. Satheesan, S. Tamgadge, A. Tamgadge, S. Bhalerao, and T. Periera, "Histopathological and radiographic analysis of dental follicle of impacted teeth using modified Gallego's stain," Journal of Clinical and Diagnostic Research, vol. 10, no. 5, pp. ZC106-ZC111, 2016.

[16] S. Adaki, B. Yashodadevi, S. Sujatha, S. Santana, N. Rakesh, and R. Adaki, "Incidence of cystic changes in impacted lower third molar," Indian Journal of Dental Research, vol. 24, no. 2, p. 183, 2013.

[17] K. Knutsson, B. Brehmer, L. Lysell, and M. Rohlin, "Pathoses associated with mandibular third molars subjected to removal,"
Oral Surgery, Oral Medicine, Oral Pathology, Oral Radiology, and Endodontology, vol. 82, no. 1, pp. 10-17, 1996.

[18] T. D. Daley, G. P. Wysocki, and G. A. Pringle, "Relative incidence of odontogenic tumors and oral and jaw cysts in a Canadian population," Oral Surgery, Oral Medicine, Oral Pathology, vol. 77, no. 3, pp. 276-280, 1994.

[19] A. A. Tambuwala, R. G. Oswal, R. S. Desale et al., "An evaluation of pathologic changes in the follicle of impacted mandibular third molars," Journal of International Oral Health, vol. 7, no. 4, pp. 58-62, 2015. 


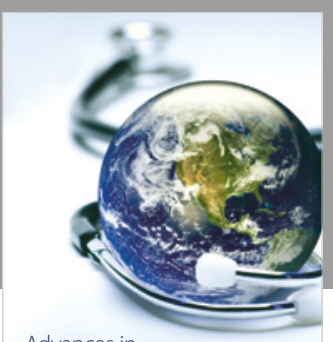

Advances in
Public Health

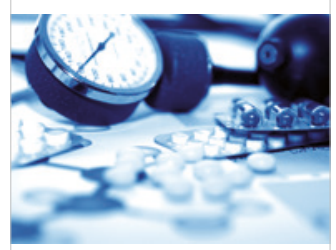

Case Reports in

Medicine

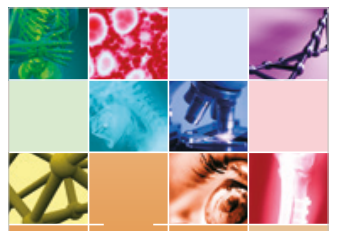

niernational Journal of

Biomaterials
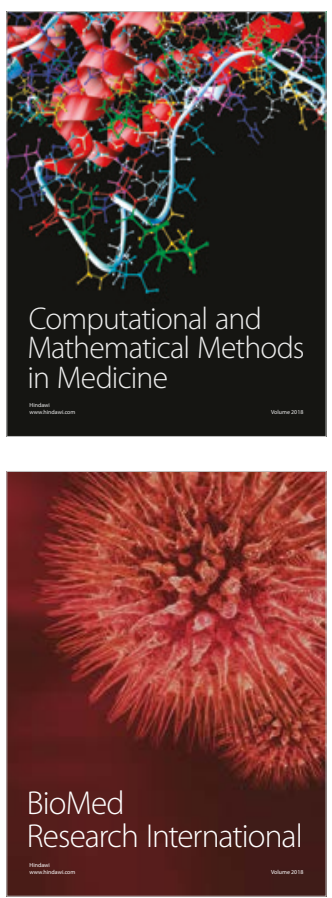

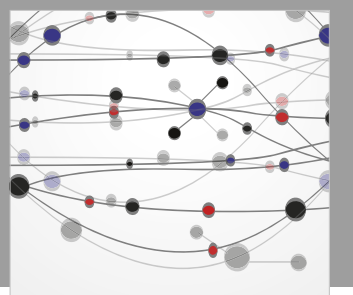

The Scientific World Journal Dentistry

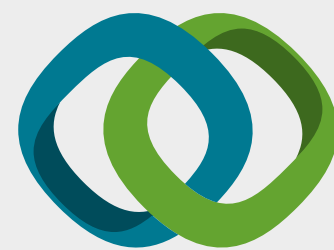

Hindawi

Submit your manuscripts at

www.hindawi.com
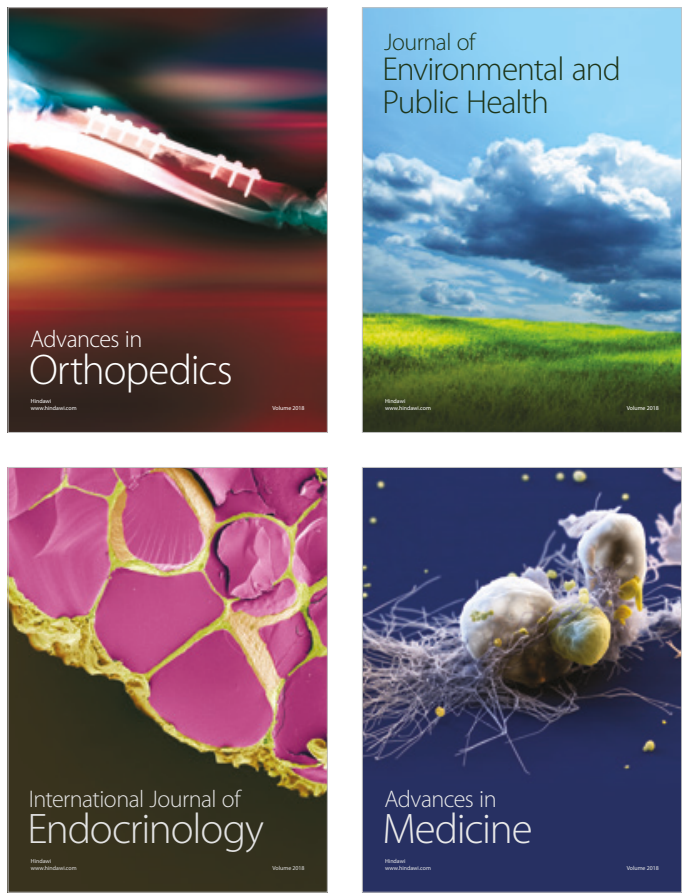
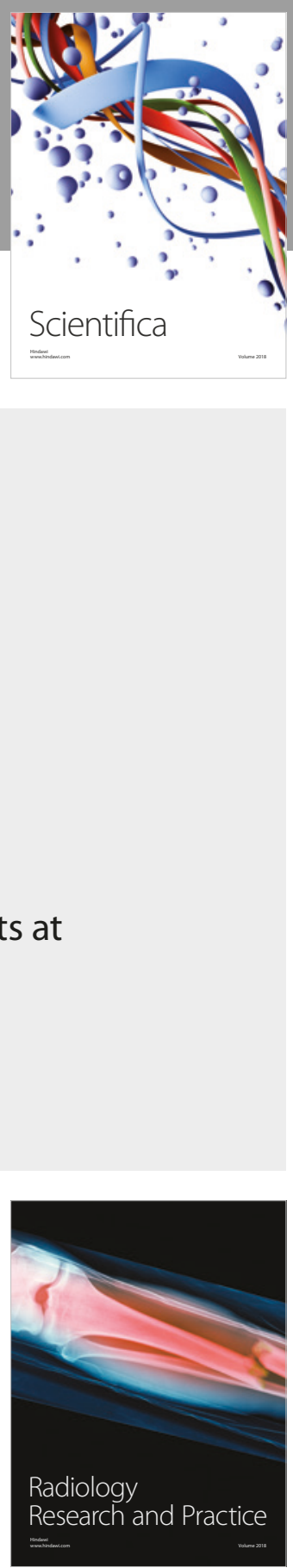

Scientifica

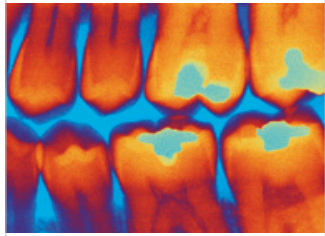

Case Reports in

Dentistry
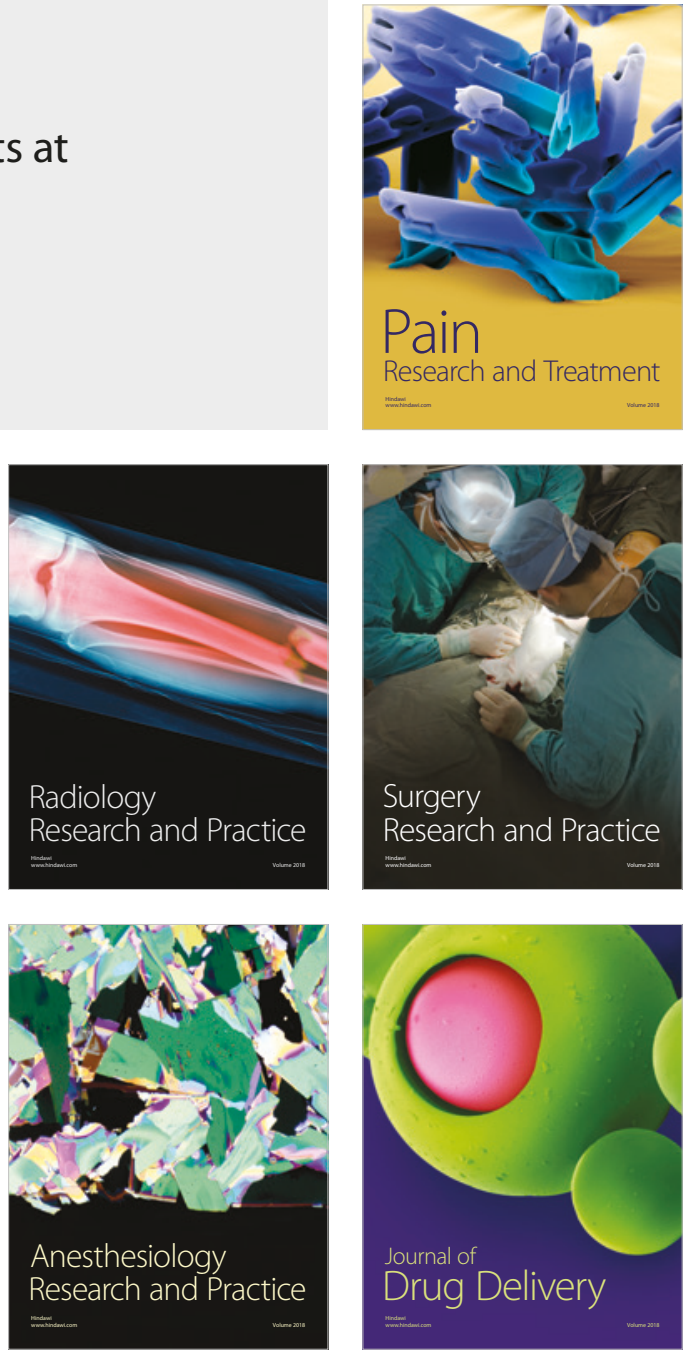